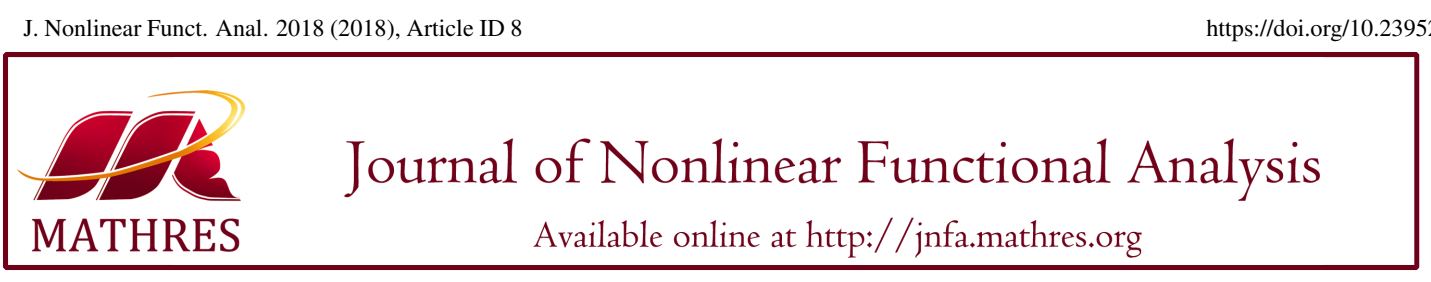

https://doi.org/10.23952/jnfa.2018.8

\title{
INFINITELY MANY SOLUTIONS FOR $2 n$-TH-ORDER BOUNDARY-VALUE PROBLEMS DEPENDING ON TWO PARAMETERS
}

\author{
OSMAN HALAKOO ${ }^{1}$, GHASEM A. AFROUZI $^{2, *}$, MAHDI AZHINI $^{1}$ \\ ${ }^{1}$ Department of Mathematics, Science and Research Branch, Islamic Azad University, Tehran, Iran \\ ${ }^{2}$ Department of Mathematics, Faculty of Mathematical Sciences, University of Mazandaran, Babolsar, Iran
}

Abstract. In this paper, using Ricceri's Variational Principle, we prove the existence of infinitely many weak solutions for a $2 n$-th-order boundary-value problem. A particular case and three concrete examples are provided to illustrate our main results. Keywords. Critical point; Boundary value problem; Infinitely many solution; Sobolev space; Variational method.

2010 Mathematics Subject Classification. 35D30, 35J65.

\section{INTRODUCTION}

Let $n \in \mathbb{N}-\{1\}$. We consider the $2 n$-th-order boundary-value problem

$$
\begin{cases}{\left[(-1)^{n} u^{(2 n)}+(-1)^{n-1} u^{(2 n-2)}+\cdots+u^{(4)}\right] h\left(x, u^{\prime}\right)-u^{\prime \prime}+u h\left(x, u^{\prime}\right)} & \\ =[\lambda f(x, u)+\mu g(x, u)+p(u)] h\left(x, u^{\prime}\right), & x \in(0,1), \\ u(0)=u(1)=u^{\prime}(0)=u^{\prime}(1)=\cdots=u^{(n-2)}(0)=u^{(n-2)}(1)=0=u^{(n)}(0)=u^{(n)}(1), & \end{cases}
$$

where $\lambda$ is a positive parameter, $\mu$ is a nonnegative parameter, $f, g:[0,1] \times \mathbb{R} \rightarrow \mathbb{R}$ are two $L^{1}$-Carathéodory functions, $p: \mathbb{R} \rightarrow(-\infty, 0]$ is a Lipschitz continuous function with the Lipschitz constant $L>0$, i.e., $\left|p\left(t_{1}\right)-p\left(t_{2}\right)\right| \leq L\left|t_{1}-t_{2}\right|$ for every $t_{1}, t_{2} \in \mathbb{R}$ with $p(0)=0$. Suppose that the Lipschitz constant $L$ of function $p$ satisfies $1 \leq L<\pi^{4}+1$, and $h:[0,1] \times \mathbb{R} \rightarrow[0,+\infty)$ is a bounded and continuous function with $0<m=\inf _{(x, t) \in[0,1] \times \mathbb{R}} h(x, t) \leq h(x, t) \leq \sup _{(x, t) \in[0,1] \times \mathbb{R}} h(x, t)=M<\infty$.

Many researchers have studied the existence and multiplicity of solutions for boundary value problem with nonlinear derivative dependence; see [1, 2, 3, 4, 5, 6, 7] and the references therein. In [7], Livrea using a three critical point theorem due to Ricceri investigated the existence of at least three classical

${ }^{*}$ Corresponding author.

E-mail addresses: osman.halakoo@ srbiau.ac.ir (O. Halakoo), afrouzi@ umz.ac.ir (G.A. Afrouzi), m.azhini@ srbiau.ac.ir (M. Azhini).

Received June 19, 2017; Accepted February 9, 2018.

(C) 2018 Journal of Nonlinear Functional Analysis 
solutions for the problem

$$
\left\{\begin{array}{l}
u^{\prime \prime}+\lambda f(x, u) h\left(u^{\prime}\right)=0, \quad x \in(0,1), \\
u(0)=u(1)=0 .
\end{array}\right.
$$

In [1], Afrouzi, Hadjian and Heidarkhani studied the existence of at least one non-trivial weak solution for the problem

$$
\left\{\begin{array}{l}
-u^{\prime \prime}=[\lambda f(x, u)+g(u)] h\left(x, u^{\prime}\right), \quad x \in(0,1), \\
u(0)=u(1)=0
\end{array}\right.
$$

where $\lambda>0$, and $f, g$ and $h$ are continuous functions with suitable conditions. In [3], Afrouzi and Shokooh studied the existence of at least three weak solutions for the problem

$$
\left\{\begin{array}{l}
u^{\prime \prime \prime \prime} h\left(x, u^{\prime}\right)-u^{\prime \prime}=[\lambda f(x, u)+g(u)] h\left(x, u^{\prime}\right), \quad x \in(0,1) \\
u(0)=u(1)=0=u^{\prime \prime}(0)=u^{\prime \prime}(1)
\end{array}\right.
$$

where $\lambda>0$, and $f, g$ and $h$ are continuous functions with convenient conditions. In [8], Afrouzi and Hadjian using Ricceri's Variational Principle (see [9, Theorem 2.1] and [10, Theorem 2.5]), established the existence of an unbounded sequence of weak solutions for the problem

$$
\left\{\begin{array}{l}
-u^{\prime \prime}+u h\left(x, u^{\prime}\right)=[\lambda f(x, u)+\mu g(x, u)+p(u)] h\left(x, u^{\prime}\right), \quad x \in(a, b), \\
u(a)=u(b)=0,
\end{array}\right.
$$

where $\lambda>0, \mu \geq 0$ and $f, g, p$ and $h$ are functions with the same conditions in problem (1.1).

In [11], Afrouzi, Kirane and Shokooh further established the existence of an unbounded sequence of weak solutions for the problem

$$
\left\{\begin{array}{l}
u^{\prime \prime \prime \prime} h\left(x, u^{\prime}\right)-u^{\prime \prime}+u h\left(x, u^{\prime}\right)=[\lambda f(x, u)+\mu g(x, u)+p(u)] h\left(x, u^{\prime}\right), \quad x \in(0,1), \\
u(0)=u(1)=0=u^{\prime \prime}(0)=u^{\prime \prime}(1),
\end{array}\right.
$$

where $\lambda>0, \mu \geq 0$ and $f, g, p$ and $h$ are functions with the same conditions in e problem (1.1).

On the other hand, Halakoo, Afrouzi and Azhini [12], employing Ricceri's Variational Principle [13], established the existence of at least three weak solutions for problem (1.2).

In this article, we investigate the existence of infinitely many solutions of problem (1.1). More precisely, under appropriate hypotheses on the nonlinear term $f, g, p$ and $h$ the existence of two interval $\Lambda$ and $J$ such that, for each $\lambda \in \Lambda$ and $\mu \in J$, the boundary value problem (1.1) admits a sequence of pairwise distinct solutions is proved (see Theorems 3.1 and 3.7). Our analysis is mainly based on a recent critical points theorem of Bonanno and Molica Bisci [9] and is contained in Theorem 2.1 below. This theorem and its variations have been used in several works in order to obtain existence results for different kinds of problems (see, for instance, [14, 15, 16, 17, 18, 19, 20, 21] and references therein). The outline of the paper is organized as follows. In Section 2, we shall recall our main tool (Theorem 2.1) and necessary basic notations which we need in the proofs. Section 3 is devoted to the existence of infinitely many weak solutions for problem (1.1). We establish our main two existence results. Some remarks and examples are also provided to illustrate our main results presented in this section. 


\section{PRELIMINARIES}

We shall prove our results applying the following smooth version of Theorem 2.1 of [9], which is a more precise version of Ricceri's Variational Principle [10, Theorem 2.5].

Theorem 2.1. Let $X$ be a reflexive real Banach space. Let $\Phi, \Psi: X \rightarrow \mathbb{R}$ be two Gâteaux differentiable functionals such that $\Phi$ is sequentially weakly lower semicontinuous, strongly continuous and coercive, and $\Psi$ is sequentially weakly upper semicontinuous. For every $r>\inf _{u \in X} \Phi(u)$, let

$$
\begin{aligned}
& \varphi(r):=\inf _{u \in \Phi^{-1}(-\infty, r)} \frac{\left(\sup _{v \in \Phi^{-1}(-\infty, r)} \Psi(v)\right)-\Psi(u)}{r-\Phi(u)}, \\
& \gamma:=\liminf _{r \rightarrow+\infty} \varphi(r), \text { and } \delta:=\liminf _{r \rightarrow\left(\inf _{X} \Phi\right)^{+}} \varphi(r) .
\end{aligned}
$$

Then

(a) for every $r>\inf _{X} \Phi$ and every $\lambda \in\left(0, \frac{1}{\varphi(r)}\right)$, the restriction of the functional $I_{\lambda}:=\Phi-\lambda \Psi$ to $\Phi^{-1}(-\infty, r)$ admits a global minimum, which is a critical point (local minimum) of $I_{\lambda}$ in $X$,

(b) if $\gamma<+\infty$, then for each $\lambda \in\left(0, \frac{1}{\gamma}\right)$, the following alternative holds: either

$\left(\mathrm{b}_{1}\right) I_{\lambda}$ possesses a global minimum, or

$\left(b_{2}\right)$ there is a sequence $\left\{u_{n}\right\}$ of critical points (local minima) of $I_{\lambda}$ such that

$$
\lim _{n \rightarrow+\infty} \Phi\left(u_{n}\right)=+\infty
$$

(c) if $\delta<+\infty$, then for each $\lambda \in\left(0, \frac{1}{\delta}\right)$, the following alternative holds: either

$\left(c_{1}\right)$ there is a global minimum of $\Phi$ which is a local minimum of $I_{\lambda}$, or

$\left(c_{2}\right)$ there is a sequence $\left\{u_{n}\right\}$ of pairwise distinct critical points (local minima) of $I_{\lambda}$ that converges weakly to a global minimum of $\Phi$.

Lemma 2.2 ([22, Lemma 2.1.3]). Let $X=W^{1,2}(a, b)$ and let $f:[a, b] \times \mathbb{R} \rightarrow \mathbb{R}$ be a $L^{1}$-Carathéodory function. For each $x \in[a, b]$ and all $t \in \mathbb{R}$, define

$$
F(x, t):=\int_{0}^{t} f(x, \xi) d \xi .
$$

Then the functional $\Psi: X \rightarrow \mathbb{R}$ given by $\Psi(u):=\int_{a}^{b} F(x, u(x)) d x$, is sequentially weakly continuous and Gâteaux differentiable on $X$ whose Gâteaux derivative is compact and we have

$$
\Psi^{\prime}(u)(v)=\int_{a}^{b} f(x, u(x)) v(x) d x, \quad \forall v \in X .
$$

Let us introduce some notations which will be used later. Define

$$
\begin{aligned}
H^{n}([0,1]):= & \left\{u \in L^{2}([0,1]): u^{\prime}, u^{\prime \prime}, \ldots, u^{(n)} \in L^{2}([0,1])\right\} \\
H_{0}^{n-1}([0,1]):= & \left\{u \in L^{2}([0,1]): u^{\prime}, u^{\prime \prime}, \ldots, u^{(n-1)} \in L^{2}([0,1])\right. \\
& \left.u(0)=u(1)=u^{\prime}(0)=u^{\prime}(1)=\cdots=u^{(n-2)}(0)=u^{(n-2)}(1)=0\right\} .
\end{aligned}
$$


Take $X=H^{n}([0,1]) \cap H_{0}^{n-1}([0,1])=\left\{u \in L^{2}([0,1]): u^{\prime}, u^{\prime \prime}, \ldots, u^{(n)} \in L^{2}([0,1])\right.$, $\left.u(0)=u(1)=u^{\prime}(0)=u^{\prime}(1)=\cdots=u^{(n-2)}(0)=u^{(n-2)}(1)=0\right\}$, endowed with the norm

$$
\|\| u \|:=\left(\left\|u^{\prime \prime}\right\|_{2}^{2}+\left\|u^{\prime \prime \prime}\right\|_{2}^{2}+\cdots+\left\|u^{(n)}\right\|_{2}^{2}\right)^{\frac{1}{2}}, \quad \text { where }\|u\|_{2}:=\left(\int_{0}^{1}|u(x)|^{2} d x\right)^{\frac{1}{2}} .
$$

We recall the following Poincaré type inequalities ([23, Lemma 2.3]):

$$
\begin{aligned}
\|u\|_{2} & \leq \frac{1}{\pi^{2}}\left\|u^{\prime \prime}\right\|_{2}, \\
\left\|u^{\prime}\right\|_{2} & \leq \frac{1}{\pi}\left\|u^{\prime \prime}\right\|_{2},
\end{aligned}
$$

for all $u \in X$. For the norm in $C^{n-1}([0,1])$,

$$
\|u\|_{\infty}:=\max \left\{\max _{x \in[0,1]}|u(x)|, \max _{x \in[0,1]}\left|u^{\prime}(x)\right|, \cdots, \max _{x \in[0,1]}\left|u^{(n-1)}(x)\right|\right\}
$$

since $C^{n-1}([0,1]) \subseteq C^{1}([0,1])$, we have the well- known inequality ([24]): $\|u\|_{\infty} \leq \frac{1}{2}\left\|u^{\prime}\right\|_{2}$. By (2.2), we have

$$
\max _{x \in[0,1]}|u(x)| \leq\|u\|_{\infty} \leq \frac{1}{2 \pi}\left\|u^{\prime \prime}\right\|_{2} \leq \frac{1}{2 \pi} \mid\|u\| \|, \quad \forall u \in X
$$

The norm ||$|\cdot|||$ is equivalent with the usual norm of Sobolev space $H^{n}((0,1))=W^{n, 2}((0,1)):\|u\|_{W^{n, 2}}=$ $\left(\|u\|_{2}^{2}+\left\|u^{\prime}\right\|_{2}^{2}+\left\|u^{\prime \prime}\right\|_{2}^{2}+\cdots+\left\|u^{(n)}\right\|_{2}^{2}\right)^{\frac{1}{2}}$. By (2.1) and (2.2) we have

$$
\begin{aligned}
\|\| u \| & =\left(\left\|u^{\prime \prime}\right\|_{2}^{2}+\left\|u^{\prime \prime \prime}\right\|_{2}^{2}+\cdots+\left\|u^{(n)}\right\|_{2}^{2}\right)^{\frac{1}{2}} \\
& \leq\left(\|u\|_{2}^{2}+\left\|u^{\prime}\right\|_{2}^{2}+\left\|u^{\prime \prime}\right\|_{2}^{2}+\cdots+\left\|u^{(n)}\right\|_{2}^{2}\right)^{\frac{1}{2}} \\
& \leq\left(\frac{1}{\pi^{4}}\left\|u^{\prime \prime}\right\|_{2}^{2}+\frac{1}{\pi^{2}}\left\|u^{\prime \prime}\right\|_{2}^{2}+\left\|u^{\prime \prime}\right\|_{2}^{2}+\cdots+\left\|u^{(n)}\right\|_{2}^{2}\right)^{\frac{1}{2}} \\
& \leq\left(\frac{1}{\pi^{4}}+\frac{1}{\pi^{2}}+1\right)^{\frac{1}{2}}\left(\left\|u^{\prime \prime}\right\|_{2}^{2}+\cdots+\left\|u^{(n)}\right\|_{2}^{2}\right)^{\frac{1}{2}} \\
& =\left(\frac{1}{\pi^{4}}+\frac{1}{\pi^{2}}+1\right)^{\frac{1}{2}}\|u\| .
\end{aligned}
$$

We recall that $f:[0,1] \times \mathbb{R} \rightarrow \mathbb{R}$ is a Carathéodory function if

(a) the mapping $x \mapsto f(x, t)$ is measurable for every $t \in \mathbb{R}$;

(b) the mapping $t \mapsto f(x, t)$ is continuous for almost every $x \in[0,1]$.

Also if for every $\rho>0$ there exists a function $\ell_{\rho} \in L^{1}([0,1])$ such that

$$
\sup _{|t| \leq \rho}|f(x, t)| \leq \ell_{\rho}(x)
$$


for almost every $x \in[0,1]$, then the Carathéodory function $f$ is called $L^{1}$-Carathéodory function. Corresponding to $f, g, p$ and $h$, we introduce the functions $F, G, P$ and $H$, respectively, as follows.

$$
\begin{array}{rlrl}
F:[0,1] \times \mathbb{R} & \rightarrow \mathbb{R} \\
(x, t) & \mapsto F(x, t):=\int_{0}^{t} f(x, \zeta) d \zeta, & G:[0,1] \times \mathbb{R} & \rightarrow \mathbb{R} \\
(x, t) & \mapsto G(x, t):=\int_{0}^{t} g(x, \zeta) d \zeta, \\
P: \mathbb{R} \rightarrow[0,+\infty) & H:[0,1] \times \mathbb{R} & \rightarrow[0,+\infty) \\
t & \mapsto P(t):=-\int_{0}^{t} p(\zeta) d \zeta, & (x, t) & \mapsto H(x, t):=\int_{0}^{t}\left(\int_{0}^{\tau} \frac{1}{h(x, \delta)} d \delta\right) d \tau,
\end{array}
$$

for all $x \in[0,1]$ and $t \in \mathbb{R}$.

If the parts of equation in (1.1) divided by $h\left(x, u^{\prime}\right)$ and multiplied by an arbitrary function $v \in X$ and then integrated in $x \in[0,1]$, then by $n$ times integration by parts we have

$$
\begin{aligned}
& \int_{0}^{1} u^{(n)}(x) v^{(n)}(x) d x+\int_{0}^{1} u^{(n-1)}(x) v^{(n-1)}(x) d x+\cdots+\int_{0}^{1} u^{\prime \prime}(x) v^{\prime \prime}(x) d x \\
& +\int_{0}^{1}\left(\int_{0}^{u^{\prime}(x)} \frac{1}{h(x, \tau)} d \tau\right) v^{\prime}(x) d x-\lambda \int_{0}^{1} f(x, u(x)) v(x) d x \\
& -\mu \int_{0}^{1} g(x, u(x)) v(x) d x-\int_{0}^{1} p(u(x)) v(x) d x=0
\end{aligned}
$$

for all $v \in X$. Then we say that function $u \in X$ in (2.4) is a weak solution of (1.1).

\section{MAIN RESULTS}

Let $0<\alpha<\beta<1$. Put

$$
\begin{gathered}
k_{1}:=\frac{\pi^{2}+m\left(\pi^{4}+1+L\right)}{2 m \pi^{4}}, \\
k_{2}:=\frac{\pi^{4}+1-L}{\pi^{4}}, \\
A:=\liminf _{\zeta \rightarrow+\infty} \frac{\int_{0}^{1} \sup _{|t| \leq \zeta} F(x, t) d x}{\zeta^{2}},
\end{gathered}
$$

and

$$
B:=\limsup _{\zeta \rightarrow+\infty} \frac{\int_{\alpha}^{\beta} F(x, \zeta) d x}{\zeta^{2}} .
$$

We formulate our main result as follows.

Theorem 3.1. Assume that there exist two positive constants $\alpha$ and $\beta$ with $0<\alpha<\beta<1$ such that

$\left(A_{1}\right) F(x, t) \geq 0$ for each $(x, t) \in([0, \alpha] \cup[\beta, 1]) \times \mathbb{R}$;

$\left(A_{2}\right)$

$$
A<\frac{2 k_{2} \pi^{2} B}{(n-1)\left(\frac{(2 n-2) !}{(n-2) !}\right)^{2}\left[\frac{1}{\alpha^{2 n-1}}+\frac{1}{(1-\beta)^{2 n-1}}\right] k_{1}}
$$

Then, setting

$$
\lambda_{1}:=\frac{(n-1)\left(\frac{(2 n-2) !}{(n-2) !}\right)^{2}\left[\frac{1}{\alpha^{2 n-1}}+\frac{1}{(1-\beta)^{2 n-1}}\right] k_{1}}{B},
$$


$\lambda_{2}:=\frac{2 k_{2} \pi^{2}}{A}$, for each $\lambda \in\left(\lambda_{1}, \lambda_{2}\right)$ and for every arbitrary $L^{1}$-Carathéodory function $g:[0,1] \times \mathbb{R} \rightarrow \mathbb{R}$, whose potential $G(x, t):=\int_{0}^{t} g(x, \zeta) d \zeta$ for all $(x, t) \in[0,1] \times \mathbb{R}$, is a nonnegative function satisfying the condition

$$
g_{\infty}:=\lim _{\zeta \rightarrow+\infty} \frac{\int_{0}^{1} \sup _{|t| \leq \zeta} G(x, t) d x}{\zeta^{2}}<+\infty .
$$

If we put

$$
\mu_{g, \lambda}:=\frac{2 k_{2} \pi^{2}}{g_{\infty}}\left(1-\lambda \frac{A}{2 k_{2} \pi^{2}}\right)
$$

where $\mu_{g, \lambda}=+\infty$ when $g_{\infty}=0$, for every $\mu \in\left[0, \mu_{g, \lambda}\right)$, problem (1.1) has an unbounded sequence of weak solutions in $X$.

Proof. Our aim is to apply Theorem (2.1) (b) to our problem. To this end, fix $\bar{\lambda} \in\left(\lambda_{1}, \lambda_{2}\right)$ and let $g$ be a function that satisfies condition (3.1). Since $\bar{\lambda}<\lambda_{2}$, we have

$$
\mu_{g, \bar{\lambda}}:=\frac{2 k_{2} \pi^{2}}{g_{\infty}}\left(1-\bar{\lambda} \frac{A}{2 k_{2} \pi^{2}}\right)>0
$$

Now fix $\bar{\mu} \in\left(0, \mu_{g, \bar{\lambda}}\right)$ and set $J(x, \zeta):=F(x, \zeta)+\frac{\bar{\mu}}{\bar{\lambda}} G(x, \zeta)$ for all $(x, \zeta) \in[0,1] \times \mathbb{R}$. We introduce the following functionals

$$
\begin{aligned}
\Phi: X & \rightarrow \mathbb{R} \\
u & \mapsto \Phi(u):=\frac{1}{2}|||u| \|^{2}+\int_{0}^{1} H\left(x, u^{\prime}(x)\right) d x+\frac{1}{2} \int_{0}^{1}|u(x)|^{2} d x+\int_{0}^{1} P(u(x)) d x, \\
\Psi: X & \rightarrow \mathbb{R} \\
u & \mapsto \Psi(u):=\int_{0}^{1} J(x, u(x)) d x,
\end{aligned}
$$

and put $I_{\bar{\lambda}}(u):=\Phi(u)-\bar{\lambda} \Psi(u), u \in X$. Note that the weak solutions of (1.1) are exactly the critical points of $I_{\bar{\lambda}}$. The functionals $\Phi$ and $\Psi$ satisfy the regularity assumptions of Theorem 2.1. Indeed, since $X$ is a reflexive real Banach space and $X$ is compactly embedded into $C([0,1])$ (note that every norm in Banach space $X$, is a sequentially weakly lower semicontinuous functional), we have that $\Phi$ is sequentially weakly lower semicontinuous and Gâteaux differentiable and its Gâteaux derivative is the functional $\Phi^{\prime}(u) \in X^{*}$, given by

$$
\begin{aligned}
& \Phi^{\prime}(u)(v)=\int_{0}^{1} u^{(n)}(x) v^{(n)}(x) d x+\cdots+\int_{0}^{1} u^{\prime \prime}(x) v^{\prime \prime}(x) d x \\
& +\int_{0}^{1}\left(\int_{0}^{u^{\prime}(x)} \frac{1}{h(x, \tau)} d \tau\right) v^{\prime}(x) d x+\int_{0}^{1} u(x) v(x) d x-\int_{0}^{1} p(u(x)) v(x) d x
\end{aligned}
$$


for every $v \in X$. Furthermore, the differential $\Phi^{\prime}: X \rightarrow X^{*}$ is a Lipschitzian operator. Indeed, by Hölder inequality, (2.1) and (2.2), for any $u, v \in X$, there holds

$$
\begin{aligned}
& \left.\left\|\Phi^{\prime}(u)-\Phi^{\prime}(v)\right\|_{X^{*}}=\sup _{\||w|\| \leq 1} \mid\left(\Phi^{\prime}(u)-\Phi^{\prime}(v)\right), w\right)\left|\leq \sup _{\|\mid\| w \| \leq 1} \int_{0}^{1}\right| u^{(n)}(x)-v^{(n)}(x) \| w^{(n)}(x) \mid d x \\
& +\cdots+\sup _{\|\mid w\| \leq 1} \int_{0}^{1}\left|u^{\prime \prime}(x)-v^{\prime \prime}(x) \| w^{\prime \prime}(x)\right| d x+\sup _{\||| w \mid\| \leq 1} \int_{0}^{1}\left|\int_{v^{\prime}(x)}^{u^{\prime}(x)} \frac{1}{h(x, \tau)} d \tau\right|\left|w^{\prime}(x)\right| d x \\
& +\sup _{\|\mid w\| \leq 1} \int_{0}^{1}\left|u(x)-v(x)\left\|w(x) d x+\sup _{\||w|\| \leq 1} \int_{0}^{1}|p(u(x))-p(v(x)) \| w(x)| d x\right.\right. \\
& \leq \sup _{\|\mid\| w \| \leq 1}\left\|u^{(n)}-v^{(n)}\right\|_{2}\left\|w^{(n)}\right\|_{2}+\cdots+\sup _{\|w\| \leq 1}\left\|u^{\prime \prime}-v^{\prime \prime}\right\|_{2}\left\|w^{\prime \prime}\right\|_{2} \\
& +\frac{1}{m} \sup _{\|w\| \leq 1}\left\|u^{\prime}-v^{\prime}\right\|_{2}\left\|w^{\prime}\right\|_{2}+(1+L) \sup _{\|w\| \leq 1}\|u-v\|_{2}\|w\|_{2} \\
& \leq\left\|u^{(n)}-v^{(n)}\right\|_{2}+\cdots+\left\|u^{\prime \prime}-v^{\prime \prime}\right\|_{2}+\frac{1}{m \pi^{2}}\left\|u^{\prime \prime}-v^{\prime \prime}\right\|_{2}+\frac{1+L}{\pi^{4}}\left\|u^{\prime \prime}-v^{\prime \prime}\right\|_{2} \\
& \leq\left(1+\frac{1}{m \pi^{2}}+\frac{1+L}{\pi^{4}}\right)\left(\left\|u^{(n)}-v^{(n)}\right\|_{2}+\cdots+\left\|u^{\prime \prime}-v^{\prime \prime}\right\|_{2}\right) \text {. }
\end{aligned}
$$

Note the fact that for every $u \in W^{n, 2}(\Omega)$ the following norms are equivalent

$$
\left\{\begin{array}{l}
\|\| u \|_{1}=\left(\|u\|_{2}+\left\|u^{\prime}\right\|_{2}+\cdots+\left\|u^{(n)}\right\|_{2}\right), \\
\|u\|_{2}=\left(\|u\|_{2}^{2}+\left\|u^{\prime}\right\|_{2}^{2}+\cdots+\left\|u^{(n)}\right\|_{2}^{2}\right)^{\frac{1}{2}}
\end{array}\right.
$$

So $u^{\prime \prime} \in W^{n-2,2}(\Omega)$. The following norms are also equivalent

$$
\left\{\begin{array}{l}
\|\| u\|\|_{3}=\left(\left\|u^{\prime \prime}\right\|_{2}+\cdots+\left\|u^{(n)}\right\|_{2}\right) \\
\|\| u\|\|_{4}=\left(\left\|u^{\prime \prime}\right\|_{2}^{2}+\cdots+\left\|u^{(n)}\right\|_{2}^{2}\right)^{\frac{1}{2}} .
\end{array}\right.
$$

Therefore, there exist two constants $c_{1}=1$ and $c_{2} \geq 1$ such that

$$
\begin{aligned}
& c_{1}\left|\|u\|\left\|_{4} \leq\right\|\|u\|_{3} \leq c_{2}\right|\|u\| \|_{4} \\
\Rightarrow & \sqrt{\left\|u^{\prime \prime}\right\|_{2}^{2}+\cdots+\left\|u^{(n)}\right\|_{2}^{2}} \leq\left(\left\|u^{\prime \prime}\right\|_{2}+\cdots+\left\|u^{(n)}\right\|_{2}\right) \leq c_{2} \sqrt{\left\|u^{\prime \prime}\right\|_{2}^{2}+\cdots+\left\|u^{(n)}\right\|_{2}^{2}} .
\end{aligned}
$$

It is necessary to say that if $n=2$, then $c_{2}=1$, i.e., if $n=2$, then $\||u|\|_{3}=\|\| u \|\left.\right|_{4}$. So we obtain from (3.2) that

$$
\begin{aligned}
\left\|\Phi^{\prime}(u)-\Phi^{\prime}(v)\right\|_{X^{*}} & \leq\left(1+\frac{1}{m \pi^{2}}+\frac{1+L}{\pi^{4}}\right)\left(\left\|u^{(n)}-v^{(n)}\right\|_{2}+\cdots+\left\|u^{\prime \prime}-v^{\prime \prime}\right\|_{2}\right) \\
& \leq 2 k_{1} c_{2}\left(\left\|u^{(n)}-v^{(n)}\right\|_{2}^{2}+\cdots+\left\|u^{\prime \prime}-v^{\prime \prime}\right\|_{2}^{2}\right)^{\frac{1}{2}}=2 k_{1} c_{2}\|\| u-v\|\| .
\end{aligned}
$$

Recalling that $p$ is Lipschitz continuous, $h$ is bounded away from zero, and the embedding $X \hookrightarrow L^{2}([0,1])$ is compact, the claim is true. In particular, we derive that $\Phi$ is continuously differentiable. On the other 
hand, for any $u, v \in X$, we have

$$
\begin{aligned}
& \left(\Phi^{\prime}(u)-\Phi^{\prime}(v), u-v\right)=\int_{0}^{1}\left(u^{(n)}(x)-v^{(n)}(x)\right)^{2} d x+\cdots+\int_{0}^{1}\left(u^{\prime \prime}(x)-v^{\prime \prime}(x)\right)^{2} d x \\
& +\int_{0}^{1}\left(\int_{v^{\prime}(x)}^{u^{\prime}(x)} \frac{1}{h(x, \tau)} d \tau\right)\left(u^{\prime}(x)-v^{\prime}(x)\right) d x+\int_{0}^{1}(u(x)-v(x))^{2} d x \\
& -\int_{0}^{1}(p(u)-p(v))(u(x)-v(x)) d x \\
& \geq\left\|u^{(n)}-v^{(n)}\right\|_{2}^{2}+\cdots+\left\|u^{\prime \prime}-v^{\prime \prime}\right\|_{2}^{2}+\frac{1}{M}\left\|u^{\prime}-v^{\prime}\right\|_{2}^{2}+(1-L)\|u-v\|_{2}^{2} \\
& \geq \mid\|u-v\|\left\|^{2}+(1-L)\right\| u-v\left\|_{2}^{2} \geq\right\|\|u-v\|\left\|^{2}+\frac{1-L}{\pi^{4}}\right\| u^{\prime \prime}-v^{\prime \prime} \|_{2}^{2} \\
& \geq\left(1+\frac{1-L}{\pi^{4}}\right)\|u-v\|\left\|^{2}=k_{2}\left|\|u-v \mid\|^{2} .\right.\right.
\end{aligned}
$$

By the assumption $1 \leq L<\pi^{4}+1$, it turns out that $\Phi^{\prime}$ is a strongly monotone operator. So, by applying Minty- Browder theorem [25, Theorem 26.A], $\Phi^{\prime}: X \rightarrow X^{*}$ admits a Lipschitz continuous inverse. Since $p$ is Lipschitz continuous and satisfies $p(0)=0$, while $h$ is bounded away from zero, we have from (2.1) and (2.2) that

$$
\begin{aligned}
|\Phi(u)|= & \left.\left|\frac{1}{2}\right|\|u\|\right|^{2}+\int_{0}^{1} H\left(x, u^{\prime}(x)\right) d x+\frac{1}{2} \int_{0}^{1}|u(x)|^{2} d x+\int_{0}^{1} P(u(x)) d x \mid \\
= & \left.\left|\frac{1}{2}\right|\|u\|\right|^{2}+\int_{0}^{1} \int_{0}^{u^{\prime}(x)}\left(\int_{0}^{\tau} \frac{1}{h(x, \delta)} d \delta\right) d \tau d x+\frac{1}{2} \int_{0}^{1}|u(x)|^{2} d x \\
& -\int_{0}^{1}\left(\int_{0}^{u(x)} p(\zeta) d \zeta\right) d x \mid \\
& \leq \frac{1}{2}\|\mid u\|\left\|^{2}+\frac{1}{2 m} \int_{0}^{1}\left(u^{\prime}(x)\right)^{2} d x+\frac{1}{2}\right\| u \|_{2}^{2}+\frac{L}{2} \int_{0}^{1}(u(x))^{2} d x \\
& =\frac{1}{2}\|\mid u\|\left\|^{2}+\frac{1}{2 m}\right\| u^{\prime}\left\|_{2}^{2}+\frac{1+L}{2}\right\| u \|_{2}^{2} \\
\leq & \frac{1}{2}\|\mid u\|\left\|^{2}+\frac{1}{2 m \pi^{2}}\right\| u^{\prime \prime}\left\|_{2}^{2}+\frac{1+L}{2 \pi^{4}}\right\| u^{\prime \prime} \|_{2}^{2} \\
\leq & \left(\frac{1}{2}+\frac{1}{2 m \pi^{2}}+\frac{1+L}{2 \pi^{4}}\right)\left\|\left|\|u\|^{2}=k_{1}\right|\right\| u\|\|^{2} .
\end{aligned}
$$

On the other hand, we have

$$
\begin{aligned}
|\Phi(u)| & \geq \frac{1}{2}\|\| u\left\|\mid+\frac{1}{2 M} \int_{0}^{1}\left(u^{\prime}(x)\right)^{2} d x+\frac{1}{2}\right\| u \|_{2}^{2}-\frac{L}{2} \int_{0}^{1}(u(x))^{2} d x \\
& \geq \frac{1}{2}\left\|\left|u\|\|^{2}+\frac{1}{2}\|u\|_{2}^{2}-\frac{L}{2}\|u\|_{2}^{2} \geq \frac{1}{2}\right|\right\| u\left\|||+\frac{1-L}{2 \pi^{4}}\right\| u^{\prime \prime} \|_{2}^{2} \\
& \geq\left.\left(\frac{1}{2}+\frac{1-L}{2 \pi^{4}}\right)\|\| u\left\|\left.\right|^{2}=\frac{k_{2}}{2}\right\|\|u\|\right|^{2} .
\end{aligned}
$$

Since $\Phi(u) \geq 0$, we have

$$
\frac{k_{2}}{2}\|\| u\|\|^{2} \leq \Phi(u) \leq k_{1}\|\| u \|\left.\right|^{2}, \quad \forall u \in X
$$

So $\Phi$ is coercive. 
On the other hand, the fact that $X$ is compactly embedded into $C([0,1])$ and Lemma 2.2 imply that the functional $\Psi$ is well defined, continuously Gâteaux differentiable and with compact derivative, whose Gâteaux derivative is given by

$$
\Psi^{\prime}(u)(v)=\int_{0}^{1} f(x, u(x)) v(x) d x+\frac{\bar{\mu}}{\bar{\lambda}} \int_{0}^{1} g(x, u(x)) v(x) d x
$$

for every $v \in X$. Hence $\Psi$ is sequentially weakly (upper) continuous [25, Corollary 41.9]. First of all, we will show that $\bar{\lambda}<\frac{1}{\gamma}$. Hence, let $\left\{\zeta_{m}\right\}$ be a sequence of positive numbers such that $\lim _{m \rightarrow+\infty} \zeta_{m}=+\infty$ and

$$
\lim _{m \rightarrow+\infty} \frac{\int_{0}^{1} \sup _{|t| \leq \zeta_{m}} F(x, t) d x}{\zeta_{m}^{2}}=A .
$$

Put $r_{m}:=2 k_{2} \pi^{2} \zeta_{m}^{2}$ for all $m \in \mathbb{N}$. Then, for all $v \in X$ with $\Phi(v)<r_{m}$, together with (2.3) and (3.3), we have $\|v\|_{\infty} \leq \zeta_{m}$. Note that $\Phi(0)=\Psi(0)=0$. Then, for all $m \in \mathbb{N}$, by choosing $u=0$, we have

$$
\begin{aligned}
\varphi\left(r_{m}\right) & =\inf _{u \in \Phi^{-1}\left(-\infty, r_{m}\right)} \frac{\left(\sup _{v \in \Phi^{-1}\left(-\infty, r_{m}\right)} \Psi(v)\right)-\Psi(u)}{r_{m}-\Phi(u)} \\
& \leq \frac{\sup _{v \in \Phi^{-1}\left(-\infty, r_{m}\right)} \Psi(v)}{r_{m}} \\
& \leq \frac{1}{2 k_{2} \pi^{2}} \frac{\int_{0}^{1} \sup _{|t| \leq \zeta_{m}} J(x, t) d x}{\zeta_{m}^{2}} \\
& \leq \frac{1}{2 k_{2} \pi^{2}}\left[\frac{\int_{0}^{1} \sup _{|t| \leq \zeta_{m}} F(x, t) d x}{\zeta_{m}^{2}}+\frac{\bar{\mu}}{\bar{\lambda}} \frac{\int_{0}^{1} \sup _{|t| \leq \zeta_{m}} G(x, t) d x}{\zeta_{m}^{2}}\right] .
\end{aligned}
$$

Moreover, from the assumption $\left(A_{2}\right)$ and condition (3.1) we have $A<+\infty$ and $\lim _{m \rightarrow+\infty} \frac{\int_{0}^{1} \sup _{|t| \leq \zeta_{m}} G(x, t) d x}{\zeta_{m}^{2}}=g_{\infty}<+\infty$. Therefore,

$$
\gamma \leq \liminf _{m \rightarrow+\infty} \varphi\left(r_{m}\right) \leq \frac{1}{2 k_{2} \pi^{2}}\left[A+\frac{\bar{\mu}}{\bar{\lambda}} g_{\infty}\right]<+\infty
$$

The assumption $\bar{\mu} \in\left(0, \mu_{g, \bar{\lambda}}\right)$ immediately yields

$$
\gamma \leq \frac{1}{2 k_{2} \pi^{2}}\left[A+\frac{\bar{\mu}}{\bar{\lambda}} g_{\infty}\right]<\frac{1}{2 k_{2} \pi^{2}} A+\frac{1-\frac{1}{2 k_{2} \pi^{2}} \bar{\lambda} A}{\bar{\lambda}} .
$$

Hence

$$
\bar{\lambda}=\frac{1}{\frac{1}{2 k_{2} \pi^{2}} A+\frac{1-\frac{1}{2 k_{2} \pi^{2}} \bar{\lambda} A}{\bar{\lambda}}}<\frac{1}{\gamma} .
$$

Let $\bar{\lambda}$ be fixed. We claim that the functional $I_{\bar{\lambda}}$ is unbounded from below. Since

$$
\frac{1}{\bar{\lambda}}<\frac{B}{(n-1)\left(\frac{(2 n-2) !}{(n-2) !}\right)^{2}\left[\frac{1}{\alpha^{2 n-1}}+\frac{1}{(1-\beta)^{2 n-1}}\right] k_{1}},
$$

we see that there exist a sequence $\left\{\eta_{m}\right\}$ of positive numbers and $\tau>0$ such that $\lim _{m \rightarrow+\infty} \eta_{m}=+\infty$ and

$$
\frac{1}{\bar{\lambda}}<\tau<\frac{\int_{\alpha}^{\beta} F\left(x, \eta_{m}\right) d x}{(n-1)\left(\frac{(2 n-2) !}{(n-2) !}\right)^{2}\left[\frac{1}{\alpha^{2 n-1}}+\frac{1}{(1-\beta)^{2 n-1}}\right] k_{1} \eta_{m}^{2}}
$$


for each $m \in \mathbb{N}$ large enough. For all $m \in \mathbb{N}$, define

$$
w_{m}(x):= \begin{cases}\eta_{m} \sum_{i=0}^{n-1}(-1)^{n-1-i}\left(\begin{array}{c}
2 n-3-i \\
n-1-i
\end{array}\right)\left(\begin{array}{c}
2 n-2 \\
i
\end{array}\right)\left(\frac{x}{\alpha}\right)^{2 n-2-i}, & x \in[0, \alpha), \\
\eta_{m}, & x \in[\alpha, \beta], \\
\frac{\eta_{m}}{(1-\beta)^{2 n-2}}\left[\left(\begin{array}{c}
2 n-3 \\
n-1
\end{array}\right)(2 n-2) \sum_{i=0}^{2 n-3} \frac{(-1)^{n-1-i}}{2 n-2-i}\left(\begin{array}{c}
\min \{i, n-2\} \\
j=\max \{0,-n+1+i\} \\
\left(\begin{array}{c}
n-2 \\
n-2-j
\end{array}\right)\left(\begin{array}{c}
n-1 \\
n-1-i+j
\end{array}\right) \beta^{i-j}
\end{array}\right) x^{2 n-2-i}+\sum_{i=n-1}^{2 n-2}(-1)^{i}\left(\begin{array}{c}
2 n-2 \\
i
\end{array}\right) \beta^{2 n-2-i}\right], & x \in(\beta, 1] .\end{cases}
$$

For any fixed $m \in \mathbb{N}$, it is easy to see that $w_{m} \in X$ and, in particular, one has

$$
4(n-1)^{2} \eta_{m}^{2}\left[\frac{1}{\alpha^{3}}+\frac{1}{(1-\beta)^{3}}\right] \leq\left\|\left|w_{m}\right|\right\|^{2} \leq(n-1) \eta_{m}^{2}\left(\frac{(2 n-2) !}{(n-2) !}\right)^{2}\left[\frac{1}{\alpha^{2 n-1}}+\frac{1}{(1-\beta)^{2 n-1}}\right] .
$$

So

$$
\begin{aligned}
2 k_{2}(n-1)^{2} \eta_{m}^{2}\left[\frac{1}{\alpha^{3}}+\frac{1}{(1-\beta)^{3}}\right] & \leq \frac{k_{2}}{2}\left\|\left|w_{m}\right|\right\|^{2} \leq \Phi\left(w_{m}\right) \leq k_{1}\left\|\left|w_{m}\right|\right\|^{2} \\
& \leq(n-1) \eta_{m}^{2} k_{1}\left(\frac{(2 n-2) !}{(n-2) !}\right)^{2}\left[\frac{1}{\alpha^{2 n-1}}+\frac{1}{(1-\beta)^{2 n-1}}\right] .
\end{aligned}
$$

On the other hand, bearing $\left(A_{1}\right)$ in mind and since $G$ is nonnegative, we infer from the definition of $\Psi$ that

$$
\Psi\left(w_{m}\right)=\int_{0}^{1}\left[F\left(x, w_{m}(x)\right)+\frac{\bar{\mu}}{\bar{\lambda}} G\left(x, w_{m}(x)\right)\right] d x \geq \int_{\alpha}^{\beta} F\left(x, \eta_{m}\right) d x .
$$

By (3.6), (3.8) and (3.9), we have

$$
\begin{aligned}
I_{\bar{\lambda}}\left(w_{m}\right) & \leq(n-1) \eta_{m}^{2} k_{1}\left(\frac{(2 n-2) !}{(n-2) !}\right)^{2}\left[\frac{1}{\alpha^{2 n-1}}+\frac{1}{(1-\beta)^{2 n-1}}\right]-\bar{\lambda} \int_{\alpha}^{\beta} F\left(x, \eta_{m}\right) d x \\
& <(n-1) \eta_{m}^{2} k_{1}\left(\frac{(2 n-2) !}{(n-2) !}\right)^{2}\left[\frac{1}{\alpha^{2 n-1}}+\frac{1}{(1-\beta)^{2 n-1}}\right](1-\bar{\lambda} \tau)
\end{aligned}
$$

for every $m \in \mathbb{N}$ large enough. Since $\bar{\lambda} \tau>1$ and $\lim _{m \rightarrow+\infty} \eta_{m}=+\infty$, we have

$$
\lim _{m \rightarrow+\infty} I_{\bar{\lambda}}\left(w_{m}\right)=-\infty .
$$

Then, the functional $I_{\bar{\lambda}}$ is unbounded from below, and it follows that $I_{\bar{\lambda}}$ has no global minimum. Therefore, by Theorem 2.1 (b), there exists a sequence $\left\{u_{m}\right\}$ of critical points of $I_{\bar{\lambda}}$ such that $\lim _{m \rightarrow+\infty}||\left|u_{m}\right| \|=$ $+\infty$, and the conclusion is achieved.

Remark 3.2. In Theorem 3.1, the aim of taking $p$ as a nonpositive function, that is, $\Phi(u)=\frac{1}{2}\|u\| \|^{2}+$ $\int_{0}^{1} H\left(x, u^{\prime}(x)\right) d x+\frac{1}{2}\|u\|_{2}^{2}+\int_{0}^{1} P(u(x)) d x$, is nonnegative. If $p: \mathbb{R} \rightarrow \mathbb{R}$ is such that $\Phi \geq 0$, then Theorem 3.1 is satisfied.

Remark 3.3. Under the conditions $A=0$ and $B=+\infty$, we see from Theorem 3.1 that for every $\lambda>0$ and for each $\mu \in\left[0, \frac{2 k_{2} \pi^{2}}{g_{\infty}}\right)$, problem (1.1) admits a sequence of weak solutions which is unbounded in $X$. Moreover, if $g_{\infty}=0$, then the result holds for every $\lambda>0$ and $\mu \geq 0$.

The following result is a special case of Theorem 3.1 with $\mu=0$. 
Theorem 3.4. Assume that there exist two positive constants $\alpha$ and $\beta$ with $0<\alpha<\beta<1$ such that

$\left(A_{1}\right) F(x, t) \geq 0$ for each $(x, t) \in([0, \alpha] \cup[\beta, 1]) \times \mathbb{R}$;

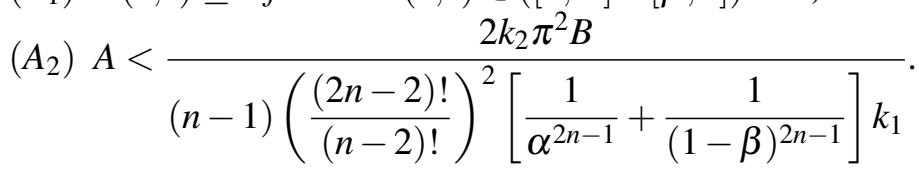

Then, for each

$$
\lambda \in\left(\frac{(n-1)\left(\frac{(2 n-2) !}{(n-2) !}\right)^{2}\left[\frac{1}{\alpha^{2 n-1}}+\frac{1}{(1-\beta)^{2 n-1}}\right] k_{1}}{B}, \frac{2 k_{2} \pi^{2}}{A}\right)
$$

the problem

$$
\begin{cases}T(u)=[\lambda f(x, u)+p(u)] h\left(x, u^{\prime}\right), & x \in(0,1), \\ u(0)=u(1)=u^{\prime}(0)=u^{\prime}(1)=\cdots=u^{(n-2)}(0)=u^{(n-2)}(1)=0=u^{(n)}(0)=u^{(n)}(1)\end{cases}
$$

where $T(u):=\left[(-1)^{n} u^{(2 n)}+(-1)^{n-1} u^{(2 n-2)}+\cdots+u^{(4)}\right] h\left(x, u^{\prime}\right)-u^{\prime \prime}+u h\left(x, u^{\prime}\right)$, has an unbounded sequence of weak solutions in $X$.

Here we point out the following consequences of Theorem 3.4.

Corollary 3.5. Assume that there exist two positive constants $\alpha$ and $\beta$ with $0<\alpha<\beta<1$ such that the assumption $\left(A_{1}\right)$ in Theorem 3.1 holds. Suppose that

$$
A<2 k_{2} \pi^{2}, \quad B>(n-1)\left(\frac{(2 n-2) !}{(n-2) !}\right)^{2}\left[\frac{1}{\alpha^{2 n-1}}+\frac{1}{(1-\beta)^{2 n-1}}\right] k_{1} .
$$

Then, the problem

$$
\left\{\begin{array}{l}
T(u)=[f(x, u)+p(u)] h\left(x, u^{\prime}\right), \quad x \in(0,1) \\
u(0)=u(1)=u^{\prime}(0)=u^{\prime}(1)=\cdots=u^{(n-2)}(0)=u^{(n-2)}(1)=0=u^{(n)}(0)=u^{(n)}(1)
\end{array}\right.
$$

where $T(u):=\left[(-1)^{n} u^{(2 n)}+(-1)^{n-1} u^{(2 n-2)}+\cdots+u^{(4)}\right] h\left(x, u^{\prime}\right)-u^{\prime \prime}+u h\left(x, u^{\prime}\right)$, has an unbounded sequence of weak solutions in $X$.

Corollary 3.6. Let $g_{1}: \mathbb{R} \rightarrow \mathbb{R}$ be a nonnegative continuous function. Put $G_{1}(\zeta):=\int_{0}^{\zeta} g_{1}(t) d t$ for all $\zeta \in \mathbb{R}$ and assume that

$$
\begin{aligned}
& \left(A_{3}\right) \liminf _{\zeta \rightarrow+\infty} \frac{G_{1}(\zeta)}{\zeta^{2}}<+\infty, \\
& \left(A_{4}\right) \limsup _{\zeta \rightarrow+\infty} \frac{G_{1}(\zeta)}{\zeta^{2}}=+\infty .
\end{aligned}
$$

Then, for every $\alpha_{i} \in L^{1}([0,1])$ for $1 \leq i \leq m$, with $\min _{x \in[0,1]}\left\{\alpha_{i}(x): 1 \leq i \leq m\right\} \geq 0$ and with $\alpha_{1} \neq 0$, and for every continuous function $g_{i}: \mathbb{R} \rightarrow \mathbb{R}$ for $2 \leq i \leq m$ satisfying

$$
\max \left\{\sup _{\zeta \in \mathbb{R}} G_{i}(\zeta): 2 \leq i \leq m\right\} \leq 0
$$

and

$$
\min \left\{\liminf _{\zeta \rightarrow+\infty} \frac{G_{i}(\zeta)}{\zeta^{2}}: 2 \leq i \leq m\right\}>-\infty
$$


where $G_{i}(\zeta):=\int_{0}^{\zeta} g_{i}(t) d t$ for all $\zeta \in \mathbb{R}$ for $2 \leq i \leq m$, for each

$$
\lambda \in\left(0, \frac{2 k_{2} \pi^{2}}{\left(\int_{0}^{1} \alpha_{1}(x) d x\right) \liminf _{\zeta \rightarrow+\infty} \frac{G_{1}(\zeta)}{\zeta^{2}}}\right)
$$

the problem

$$
\left\{\begin{array}{l}
T(u)=\left[\lambda \sum_{i=1}^{m} \alpha_{i}(x) g_{i}(u)+p(u)\right] h\left(x, u^{\prime}\right), \quad x \in(0,1), \\
u(0)=u(1)=u^{\prime}(0)=u^{\prime}(1)=\cdots=u^{(n-2)}(0)=u^{(n-2)}(1)=0=u^{(n)}(0)=u^{(n)}(1),
\end{array}\right.
$$

where $T(u):=\left[(-1)^{n} u^{(2 n)}+(-1)^{n-1} u^{(2 n-2)}+\cdots+u^{(4)}\right] h\left(x, u^{\prime}\right)-u^{\prime \prime}+u h\left(x, u^{\prime}\right)$, has an unbounded sequence of weak solutions in $X$.

Proof. Put $f(x, t)=\sum_{i=1}^{m} \alpha_{i}(x) g_{i}(t)$ for all $(x, t) \in[0,1] \times \mathbb{R}$. The assumption $\left(A_{4}\right)$ in conjunction with the condition

$$
\min \left\{\liminf _{\zeta \rightarrow+\infty} \frac{G_{i}(\zeta)}{\zeta^{2}}: 2 \leq i \leq m\right\}>-\infty
$$

yields

$$
\limsup _{\zeta \rightarrow+\infty} \frac{\int_{\alpha}^{\beta} F(x, \zeta) d x}{\zeta^{2}}=\limsup _{\zeta \rightarrow+\infty} \frac{\sum_{i=1}^{m}\left(G_{i}(\zeta) \int_{\alpha}^{\beta} \alpha_{i}(x) d x\right)}{\zeta^{2}}=+\infty .
$$

Moreover, the assumption $\left(A_{3}\right)$ along with the condition

$$
\max \left\{\sup _{\zeta \in \mathbb{R}} G_{i}(\zeta): 2 \leq i \leq m\right\} \leq 0
$$

ensures

$$
\liminf _{\zeta \rightarrow+\infty} \frac{\int_{0}^{1} \sup _{|t| \leq \zeta} F(x, t) d x}{\zeta^{2}} \leq\left(\int_{0}^{1} \alpha_{1}(x) d x\right) \liminf _{\zeta \rightarrow+\infty} \frac{G_{1}(\zeta)}{\zeta^{2}}<+\infty .
$$

Hence, the conclusion follows from Theorem 3.4.

Put

$$
A^{\prime}:=\liminf _{\zeta \rightarrow 0^{+}} \frac{\int_{0}^{1} \sup _{|t| \leq \zeta} F(x, t) d x}{\zeta^{2}}
$$

and

$$
B^{\prime}:=\limsup _{\zeta \rightarrow 0^{+}} \frac{\int_{\alpha}^{\beta} F(x, \zeta) d x}{\zeta^{2}} .
$$

Using Theorem 2.1 (c) and arguing as in the proof of Theorem 3.1, we can obtain the following result.

Theorem 3.7. Assume that there exist two constants $\alpha$ and $\beta$ with $0<\alpha<\beta<1$ such that $\left(A_{1}\right)$ in Theorem 3.1 holds and

$$
\left(A_{5}\right) A^{\prime}<\frac{2 k_{2} \pi^{2} B^{\prime}}{(n-1)\left(\frac{(2 n-2) !}{(n-2) !}\right)^{2}\left[\frac{1}{\alpha^{2 n-1}}+\frac{1}{(1-\beta)^{2 n-1}}\right] k_{1}} .
$$


Then, setting

$$
\lambda_{3}:=\frac{(n-1)\left(\frac{(2 n-2) !}{(n-2) !}\right)^{2}\left[\frac{1}{\alpha^{2 n-1}}+\frac{1}{(1-\beta)^{2 n-1}}\right] k_{1}}{B^{\prime}}, \quad \lambda_{4}:=\frac{2 k_{2} \pi^{2}}{A^{\prime}},
$$

for each $\lambda \in\left(\lambda_{3}, \lambda_{4}\right)$ and for every arbitrary $L^{1}$-Carathéodory function $g:[0,1] \times \mathbb{R} \rightarrow \mathbb{R}$, whose potential $G(x, t):=\int_{0}^{t} g(x, \zeta) d \zeta$ for all $(x, t) \in[0,1] \times \mathbb{R}$, is a nonnegative function satisfying the condition

$$
g_{0}:=\lim _{\zeta \rightarrow 0^{+}} \frac{\int_{0}^{1} \sup _{|t| \leq \zeta} G(x, t) d x}{\zeta^{2}}<+\infty .
$$

If we put

$$
\mu_{g, \lambda}^{\prime}:=\frac{2 k_{2} \pi^{2}}{g_{0}}\left(1-\lambda \frac{A^{\prime}}{2 k_{2} \pi^{2}}\right)
$$

where $\mu_{g, \lambda}^{\prime}=+\infty$ when $g_{0}=0$, for every $\mu \in\left[0, \mu_{g, \lambda}^{\prime}\right)$, problem (1.1) has a sequence of weak solutions, which strongly converges to zero in $X$.

Proof. Fix $\bar{\lambda} \in\left(\lambda_{3}, \lambda_{4}\right)$ and let $g$ be a function that satisfies the condition (3.11). Since $\bar{\lambda}<\lambda_{4}$, one has

$$
\mu_{g, \bar{\lambda}}^{\prime}=\frac{2 k_{2} \pi^{2}}{g_{0}}\left(1-\bar{\lambda} \frac{A^{\prime}}{2 k_{2} \pi^{2}}\right)>0 .
$$

Now, we fix $\bar{\mu} \in\left(0, \mu_{g, \bar{\lambda}}^{\prime}\right)$ and set

$$
J(x, \zeta):=F(x, \zeta)+\frac{\bar{\mu}}{\bar{\lambda}} G(x, \zeta)
$$

for all $(x, \zeta) \in[0,1] \times \mathbb{R}$. We take $\Phi, \Psi$ and $I_{\bar{\lambda}}$ as in the proof of Theorem 3.1. Now, as was pointed out before, the functionals $\Phi$ and $\Psi$ satisfy the regularity assumptions required in Theorem 2.1. As the first step, we will prove that $\bar{\lambda}<\frac{1}{\delta}$. Let $\left\{\zeta_{m}\right\}$ be a sequence of positive numbers such that $\lim _{m \rightarrow+\infty} \zeta_{m}=0^{+}$and

$$
\lim _{m \rightarrow+\infty} \frac{\int_{0}^{1} \sup _{|t| \leq \zeta_{m}} F(x, t) d x}{\zeta_{m}^{2}}=A^{\prime} .
$$

By the fact that $\inf _{X} \Phi=0$ and the definition of $\delta$, we have $\delta=\liminf _{r \rightarrow 0^{+}} \varphi(r)$. Then, as in showing (3.5) in the proof of Theorem 3.1, we can prove that $\delta<+\infty$. From $\bar{\mu} \in\left(0, \mu_{g, \bar{\lambda}}^{\prime}\right)$, we see that the following inequalities hold

$$
\delta \leq \frac{1}{2 k_{2} \pi^{2}}\left(A^{\prime}+\frac{\bar{\mu}}{\bar{\lambda}} g_{0}\right)<\frac{1}{2 k_{2} \pi^{2}} A^{\prime}+\frac{1-\frac{1}{2 k_{2} \pi^{2}} \bar{\lambda} A^{\prime}}{\bar{\lambda}} .
$$

Hence,

$$
\bar{\lambda}=\frac{1}{\frac{1}{2 k_{2} \pi^{2}} A^{\prime}+\frac{1-\frac{1}{2 k_{2} \pi^{2}} \bar{\lambda} A^{\prime}}{\bar{\lambda}}}<\frac{1}{\delta} .
$$

Let $\bar{\lambda}$ be fixed. We claim that the functional $I_{\bar{\lambda}}$ has not a local minimum at zero. Since

$$
\frac{1}{\bar{\lambda}}<\frac{B^{\prime}}{(n-1)\left(\frac{(2 n-2) !}{(n-2) !}\right)^{2}\left[\frac{1}{\alpha^{2 n-1}}+\frac{1}{(1-\beta)^{2 n-1}}\right] k_{1}},
$$


there exists a sequence $\left\{\eta_{m}\right\}$ of positive numbers and $\tau>0$ such that $\lim _{m \rightarrow+\infty} \eta_{m}=0^{+}$and

$$
\frac{1}{\bar{\lambda}}<\tau<\frac{1}{(n-1)\left(\frac{(2 n-2) !}{(n-2) !}\right)^{2}\left[\frac{1}{\alpha^{2 n-1}}+\frac{1}{(1-\beta)^{2 n-1}}\right] k_{1}} \frac{\int_{\alpha}^{\beta} F\left(x, \eta_{m}\right) d x}{\eta_{m}^{2}}
$$

for each $m \in \mathbb{N}$ large enough. For all $m \in \mathbb{N}$, define $w_{m}(x)$ as in (3.7) with the above $\eta_{m}$. Note that $\bar{\lambda} \tau>1$. As in showing (3.10), we can obtain that

$$
\begin{aligned}
I_{\bar{\lambda}}\left(w_{m}\right) & =\Phi\left(w_{m}\right)-\bar{\lambda} \Psi\left(w_{m}\right) \\
& \leq(n-1) \eta_{m}^{2} k_{1}\left(\frac{(2 n-2) !}{(n-2) !}\right)^{2}\left[\frac{1}{\alpha^{2 n-1}}+\frac{1}{(1-\beta)^{2 n-1}}\right]-\bar{\lambda} \int_{\alpha}^{\beta} F\left(x, \eta_{m}\right) d x \\
& <(n-1) \eta_{m}^{2} k_{1}\left(\frac{(2 n-2) !}{(n-2) !}\right)^{2}\left[\frac{1}{\alpha^{2 n-1}}+\frac{1}{(1-\beta)^{2 n-1}}\right](1-\bar{\lambda} \tau)<0
\end{aligned}
$$

for every $m \in \mathbb{N}$ large enough. Since

$$
\lim _{m \rightarrow+\infty} I_{\bar{\lambda}}\left(w_{m}\right)=I_{\bar{\lambda}}(0)=0,
$$

we see that zero is not a local minimum of $I_{\bar{\lambda}}$. This, together with the fact that zero is the only global minimum of $\Phi$, shows that the functional $I_{\bar{\lambda}}$ has not a local minimum at the unique global minimum of $\Phi$. Therefore, by Theorem 2.1 (c), there exists a sequence $\left\{u_{m}\right\}$ of critical points of $I_{\bar{\lambda}}$ which converges weakly to zero. In view of the fact that the embedding $X \hookrightarrow C([0,1])$ is compact, we know that the critical points converge strongly to zero. The proof is complete.

Remark 3.8. Under the conditions $A^{\prime}=0$ and $B^{\prime}=+\infty$, Theorem 3.7 ensures that for every $\lambda>0$ and for each $\mu \in\left[0, \frac{2 k_{2} \pi^{2}}{g_{0}}\right)$, problem (1.1) admits a sequence of weak solutions, which strongly converges to zero in $X$. Moreover, if $g_{0}=0$, the result holds for every $\lambda>0$ and $\mu \geq 0$.

Remark 3.9. Applying Theorem 3.7, results similar to Theorem 3.4, and Corollaries 3.5 and 3.6 can be obtained. So, we omit the discussions here.

We conclude this paper with the following examples to illustrate our results.

Example 3.10. Put

$$
a_{m}:=\frac{2 m !(m+2) !-1}{4(m+1) !}, \quad b_{m}:=\frac{2 m !(m+2) !+1}{4(m+1) !},
$$

for every $m \in \mathbb{N}$, and define the nonnegative continuous function $f: \mathbb{R} \rightarrow \mathbb{R}$ by

$$
f(t):= \begin{cases}\frac{32(m+1) !^{2}\left[(m+1) !^{2}-m !^{2}\right]}{\pi} \sqrt{\frac{1}{16(m+1) !^{2}}-\left(t-\frac{m !(m+2)}{2}\right)^{2}}, & t \in \bigcup_{m \in \mathbb{N}}\left[a_{m}, b_{m}\right], \\ 0, & \text { otherwise. }\end{cases}
$$

One has

$$
\int_{m !}^{(m+1) !} f(t) d t=\int_{a_{m}}^{b_{m}} f(t) d t=(m+1) !^{2}-m !^{2}
$$

for every $m \in \mathbb{N}$. It follows that

$$
\lim _{m \rightarrow+\infty} \frac{F\left(a_{m}\right)}{a_{m}^{2}}=0 \quad \text { and } \quad \lim _{m \rightarrow+\infty} \frac{F\left(b_{m}\right)}{b_{m}^{2}}=4 .
$$


Note that there is no sequence $\left\{c_{m}\right\}$ such that $\lim _{m \rightarrow+\infty} c_{m}=+\infty$ and $\lim _{m \rightarrow+\infty} \frac{F\left(c_{m}\right)}{c_{m}^{2}}>4$. Therefore,

$$
\liminf _{\zeta \rightarrow+\infty} \frac{F(\zeta)}{\zeta^{2}}=0 \quad \text { and } \quad \limsup _{\zeta \rightarrow+\infty} \frac{F(\zeta)}{\zeta^{2}}=4
$$

Hence, by choosing $0<\alpha<\beta<1, p(t)=\sin t$ for all $t \in \mathbb{R}$ and $h(x, t) \equiv 4(2+x+\cos t)^{-1}$ for all $(x, t) \in[0,1] \times \mathbb{R}$, we have $m=1, M=4, L=1, k_{1}=\frac{\pi^{4}+\pi^{2}+2}{2 \pi^{4}}$ and $k_{2}=1$. For all $n \in \mathbb{N}-\{1\}$, we have

$$
\begin{aligned}
0=A & <\frac{2 k_{2} \pi^{2} B}{(n-1)\left(\frac{(2 n-2) !}{(n-2) !}\right)^{2}\left[\frac{1}{\alpha^{2 n-1}}+\frac{1}{(1-\beta)^{2 n-1}}\right] k_{1}} \\
& =\frac{16 \pi^{6}(\beta-\alpha)}{\left(\pi^{4}+\pi^{2}+2\right)(n-1)\left(\frac{(2 n-2) !}{(n-2) !}\right)^{2}\left[\frac{1}{\alpha^{2 n-1}}+\frac{1}{(1-\beta)^{2 n-1}}\right]} .
\end{aligned}
$$

So, from Theorem 3.4, for each

$$
\lambda>\frac{\left(\pi^{4}+\pi^{2}+2\right)(n-1)\left(\frac{(2 n-2) !}{(n-2) !}\right)^{2}\left[\frac{1}{\alpha^{2 n-1}}+\frac{1}{(1-\beta)^{2 n-1}}\right]}{8 \pi^{4}(\beta-\alpha)},
$$

the problem

$$
\left\{\begin{array}{l}
(-1)^{n} u^{(2 n)}+(-1)^{n-1} u^{(2 n-2)}+\cdots+u^{(4)}-\frac{1}{4} u^{\prime \prime}\left(2+x+\cos u^{\prime}\right)+u=\lambda f(u)+\sin u, \quad x \in(0,1), \\
u(0)=u(1)=u^{\prime}(0)=u^{\prime}(1)=\cdots=u^{(n-2)}(0)=u^{(n-2)}(1)=0=u^{(n)}(0)=u^{(n)}(1),
\end{array}\right.
$$

admits a sequence of weak solutions which is unbounded in $X=H^{n}([0,1]) \cap H_{0}^{n-1}([0,1])$.

The function $f$ in the following example is coming with the aid of of [22].

Example 3.11. Consider the problem $(n \in \mathbb{N}-\{1\})$

$$
\left\{\begin{array}{l}
(-1)^{n} u^{(2 n)}+(-1)^{n-1} u^{(2 n-2)}+\cdots+u^{(4)}-u^{\prime \prime}\left(2+x+\sin u^{\prime}\right)+4 u=\lambda f(u), \quad x \in(0,1), \\
u(0)=u(1)=u^{\prime}(0)=u^{\prime}(1)=\cdots=u^{(n-2)}(0)=u^{(n-2)}(1)=0=u^{(n)}(0)=u^{(n)}(1),
\end{array}\right.
$$

where

$$
\begin{aligned}
f:[0,1] \times \mathbb{R} & \rightarrow \mathbb{R} \\
(x, t) & \mapsto f(x, t)=f(t)= \begin{cases}t[2-2 \sin (\ln |t|)-\cos (\ln |t|)], & t \neq 0, \\
0, & t=0 .\end{cases}
\end{aligned}
$$

Then $f$ is a continuous function. It follows that

$$
F(t)=\int_{0}^{t} f(x, \xi) d \xi= \begin{cases}t^{2}[1-\sin (\ln |t|)], & t \neq 0 \\ 0, & t=0\end{cases}
$$

Hence, $F(t) \geq 0, \forall t \in \mathbb{R}$. Therefore,

$$
\liminf _{\xi \rightarrow 0^{+}} \frac{\sup _{|t| \leq \xi} F(t)}{\xi^{2}}=0 \text { and } \limsup _{\xi \rightarrow 0^{+}} \frac{F(\xi)}{\xi^{2}}=2 .
$$


Hence, by choosing $0<\alpha<\beta<1, p(t)=-3 t$ for all $t \in \mathbb{R}$ and $h(x, t)=(2+x+\sin t)^{-1}$ for all $(x, t) \in[0,1] \times \mathbb{R}$, we have $m=\frac{1}{4}, M=1, L=3, k_{1}=\frac{\frac{1}{2} \pi^{4}+2 \pi^{2}+2}{\pi^{4}}$ and $k_{2}=\frac{\pi^{4}-2}{\pi^{4}}$. Then for all $n \in \mathbb{N}-\{1\}$, we have

$$
\begin{aligned}
0=A^{\prime} & <\frac{2 k_{2} \pi^{2} B^{\prime}}{(n-1)\left(\frac{(2 n-2) !}{(n-2) !}\right)^{2}\left[\frac{1}{\alpha^{2 n-1}}+\frac{1}{(1-\beta)^{2 n-1}}\right] k_{1}} \\
& =\frac{4 \pi^{2}\left(\pi^{4}-2\right)(\beta-\alpha)}{\left(\frac{1}{2} \pi^{4}+2 \pi^{2}+2\right)(n-1)\left(\frac{(2 n-2) !}{(n-2) !}\right)^{2}\left[\frac{1}{\alpha^{2 n-1}}+\frac{1}{(1-\beta)^{2 n-1}}\right]} .
\end{aligned}
$$

So, from Theorem 3.7, for each

$$
\lambda>\frac{\left(\frac{1}{2} \pi^{4}+2 \pi^{2}+2\right)(n-1)\left(\frac{(2 n-2) !}{(n-2) !}\right)^{2}\left[\frac{1}{\alpha^{2 n-1}}+\frac{1}{(1-\beta)^{2 n-1}}\right]}{2 \pi^{4}(\beta-\alpha)},
$$

the problem (3.13) has a sequence of weak solutions, which strongly converges to zero in $X=H^{n}([0,1]) \cap$ $H_{0}^{n-1}([0,1])$.

Example 3.12. Consider the problem $(n \in \mathbb{N}-\{1\})$

$$
\left\{\begin{array}{l}
(-1)^{n} u^{(2 n)}+(-1)^{n-1} u^{(2 n-2)}+\cdots+u^{(4)}-u^{\prime \prime}+2 u=\lambda f(u)+\mu g(u), \\
u(0)=u(1)=u^{\prime}(0)=u^{\prime}(1)=\cdots=u^{(n-2)}(0)=u^{(n-2)}(1)=0=u^{(n)}(0)=u^{(n)}(1),
\end{array} \quad x \in(0,1),\right.
$$

where

$$
\begin{aligned}
f:[0,1] \times \mathbb{R} & \rightarrow \mathbb{R} \\
(x, t) & \mapsto f(x, t)=f(t)= \begin{cases}0, & t \in(-\infty, 0], \\
2 t\left[7 \sin ^{2}\left(\frac{1}{7} \ln t\right)+\sin \left(\frac{1}{7} \ln t\right) \cos \left(\frac{1}{7} \ln t\right)\right], & t \in(0,+\infty),\end{cases}
\end{aligned}
$$

and

$$
\begin{aligned}
g:[0,1] \times \mathbb{R} \rightarrow \mathbb{R} \\
\qquad(x, t) \mapsto g(x, t)=g(t)= \begin{cases}0, & t \in(-\infty, 0], \\
\sin ^{2} t+t \sin (2 t), & t \in(0,+\infty) .\end{cases}
\end{aligned}
$$

Hence, $f$ and $g$ are continuous, and we have

$$
F(t)=\int_{0}^{t} f(\xi) d \xi= \begin{cases}0, & t \in(-\infty, 0] \\ 7 t^{2} \sin ^{2}\left(\frac{1}{7} \ln t\right), & t \in(0,+\infty)\end{cases}
$$

and

$$
G(t)=\int_{0}^{t} g(\xi) d \xi= \begin{cases}0, & t \in(-\infty, 0] \\ t \sin ^{2} t, & t \in(0,+\infty)\end{cases}
$$


Then $F(t) \geq 0$ and $G(t) \geq 0$, for all $t \in \mathbb{R}$. Therefore,

$$
\begin{aligned}
& \liminf _{\xi \rightarrow+\infty} \frac{\sup _{|t| \leq \xi} F(t)}{\xi^{2}}=0, \quad \limsup _{\xi \rightarrow+\infty} \frac{F(\xi)}{\xi^{2}}=7 \text { and } \\
& g_{\infty}=\lim _{\xi \rightarrow+\infty} \frac{\sup _{|t| \leq \xi} G(t)}{\xi^{2}}=0<+\infty .
\end{aligned}
$$

Hence, by choosing $0<\alpha<\beta<1, p(t)=-t$ for all $t \in \mathbb{R}$ and $h(x, t)=1$ for all $(x, t) \in[0,1] \times \mathbb{R}$, we have $m=1=M, L=1, k_{1}=\frac{\pi^{4}+\pi^{2}+2}{2 \pi^{4}}$ and $k_{2}=1$, then for all $n \in \mathbb{N}-\{1\}$, we have

$$
\begin{aligned}
0=A & <\frac{2 k_{2} \pi^{2} B}{(n-1)\left(\frac{(2 n-2) !}{(n-2) !}\right)^{2}\left[\frac{1}{\alpha^{2 n-1}}+\frac{1}{(1-\beta)^{2 n-1}}\right] k_{1}} \\
& =\frac{28 \pi^{6}(\beta-\alpha)}{\left(\pi^{4}+\pi^{2}+2\right)(n-1)\left(\frac{(2 n-2) !}{(n-2) !}\right)^{2}\left[\frac{1}{\alpha^{2 n-1}}+\frac{1}{(1-\beta)^{2 n-1}}\right]} .
\end{aligned}
$$

So, from Theorem 3.1, for each

$$
(\lambda, \mu) \in\left(\frac{\left(\pi^{4}+\pi^{2}+2\right)(n-1)\left(\frac{(2 n-2) !}{(n-2) !}\right)^{2}\left[\frac{1}{\alpha^{2 n-1}}+\frac{1}{(1-\beta)^{2 n-1}}\right]}{14 \pi^{4}(\beta-\alpha)},+\infty\right) \times[0,+\infty),
$$

the problem (3.14) has an unbounded sequence of weak solutions in $X=H^{n}([0,1]) \cap H_{0}^{n-1}([0,1])$.

\section{REFERENCES}

[1] G.A. Afrouzi, A. Hadjian, S. Heidarkhani, Non-trivial solutions for a two-point boundary value problem, Ann. Polon. Math. 108 (2013), 75-84.

[2] G.A. Afrouzi, S. Heidarkhani, Three solutions for a quasilinear boundary value problem, Nonlinear Anal. 69 (2008), 3330-3336.

[3] G.A. Afrouzi, S. Shokooh, Three solutions for a fourth-order boundary-value problem, Electron. J. differential equations 2015 (2015), Article ID 45.

[4] D. Averna, G. Bonanno, Three solutions for quasilinear two-point boundary value problem involving the one-dimensional p-Laplacian, Proc. Edinb. Math. Soc. 47 (2004), 257-270.

[5] S. Heidarkhani, Infinitely many solutions for systems of $n$ two-point Kirchhoff-type boundary value problems, Ann. Polon. Math. 107 (2013), 133-152.

[6] S. Heidarkhani, D. Motreanu, Multiplicity results for a two-point boundary value problem, PanAmer. Math. J. 19 (2009), 69-78.

[7] R. Livrea, Existence of three solutions for a quasilinear two point boundary value problem, Arch. Math. 79 (2002), 288298.

[8] G.A. Afrouzi, A. Hadjian, Infinitely many solutions for a Dirichlet boundary value problem depending on two parameters, Glas. Mat. 48 (2013), 357-371.

[9] G. Bonanno, G. Molica Bisci, Infinitely many solutions for a boundary value problem with discontinuous nonlinearities, Bound. Value Probl. 2009 (2009), 1-20.

[10] B. Ricceri, A general variational principle and some of its applications, J. Comput. Appl. Math. 113 (2000), 401-410.

[11] G.A. Afrouzi, M. Kirane, S. Shokooh, Infinitely many solutions for fourth-order boundary-value problems depending on two parameters, Submitted.

[12] O. Halakoo, G.A. Afrouzi, M. Azhini, An existence result of three solutions for a fourth-order boundary-value problem, submitted.

[13] B. Ricceri, A three critical points theorem revisited, Nonlinear Anal. 70 (2009), 3084-3089. 
[14] G.A. Afrouzi, A. Hadjian, Infinitely many solutions for a class of Dirichlet quasilinear elliptic systems, J. Math. Anal. Appl. 393 (2012), 265-272.

[15] D. Averna, S.M. Buccellato, E. Tornatore, On a mixed boundary value problem involving the $p$-Laplacian, Matematiche (Catania) 66 (2011), 93-104.

[16] G. Bonanno, G.D’Aguì, A Neumann boundary value problem for the Sturm Liouville equation, Appl. Math. Comput. 208 (2009), 318-327.

[17] G. Bonanno, G.D’Aguì, Multiplicity results for a perturbed elliptic Neumann problem, Abstr. Appl. Anal. 2010 (2010), $1-10$.

[18] G. Bonanno, B. Di Bella, Infinitely many solutions for a fourth-order elastic beam equation, NoDEA Nonlinear Differential Equations Appl. 18 (2011), 357-368.

[19] G. Bonanno, G. Molica Bisci, Infinitely many solutions for a Dirichlet problem involving the $p$-Laplacian, Proc. Roy. Soc. Edinburgh Sect. A 140 (2010), 737-752.

[20] G. Bonanno, G. Molica Bisci, D. O’Regan, Infinitely many weak solutions for a class of quasilinear elliptic systems, Math. Comput. Modelling 52 (2010), 152-160.

[21] S. Heidarkhani, M. Ferrara, A. Salari, Infinitely many periodic solutions for a class of perturbed second-order differential equations with impulses, Acta Appl. Math. 139 (2015), 81-94.

[22] A. Hadjian, Existence and multiplicity results for some elliptic boundary value problems investigated via variational methods, Ph.D. Thesis, University of Mazandaran, Babolsar, Iran, 2014.

[23] L.A. Peletier, W.C. Troy, R.C.A.M. Van der Vorst, Stationary solutions of a fourth order nonlinear diffusion equation, Differentsial'nye Uravneniya, 31 (1995), 327-337.

[24] G. Talenti, Some inequalities of Sobolev type on two-dimensional spheres, in: W. Walter (ed.), General Inequalities, Vol. 5, Int. Ser. Numer. Math. Birkhäuser, Basel 80 (1987), 401-408.

[25] E. Zeidler, Nonlinear Functional Analysis and its Applications, Vol. II/B and III, Berlin-Heidelberg-New York, 1990. 